\title{
The Future Belongs to Those Who Shape It
}

\author{
David Schwartz, DDS, D.ABDSM
}

Sleep Better Chicagoland

President-Elect of the AADSM Board of Directors

\section{This editorial has been approved by the American Academy of Dental Sleep Medicine (AADSM) Board of}

Directors.

Late last year, I had the opportunity to share my thoughts about the American Dental Association (ADA) Proposed Policy on the Role of Dentistry in the Treatment of Sleep-Related Breathing Disorders with readers of the Journal of Dental Sleep Medicine. ${ }^{1}$ This policy was eventually adopted in late October $2017 .^{2}$ Like a lot of national policies, many dentists are pleased with the outcome, many are disappointed because the policy did not go far enough, and still others are disappointed because of what the policy failed to address. The American Academy of Dental Sleep Medicine (AADSM) falls into the third category, as the ADA policy does not address the important issue of education and training of dentists desiring to expand their practice by providing oral appliance therapy for those suffering from a sleep-related breathing disorder. This is a serious concern of patients. It should also be a serious concern for dentists not appropriately trained to treat patients with OAT. The professional liability exposure is significantly higher in the medicolegal environment than in general dentistry. Providing treatment for a medical disease does not start or stop with the placement of an appliance. Most if not all patients with obstructive sleep apnea (OSA) have one or more comorbidities, and some patients die from the disease. High-quality care is an absolute must and proper education and training are the only pathways to ensure quality care.

Let's be clear - there are many dentists and lots of industry perfectly happy with "no additional training needed," and why not? They are in the business of selling oral appliances or other related services. Dentists who treat patients with a medical disorder to improve and possibly save the lives of their patients must ask themselves, "Can it be true that a serious medical disorder can be effectively treated with no additional training following the completion of dental school?" The answer is no. At this time, it is an unreasonable expectation of dental school curricula to satisfactorily educate the neophyte dentist. The inherent risk to the patient coupled with the professional liability risk the dentist is assuming is far too great to be fooled into thinking that no additional training is needed.

The AADSM applauds the ADA for undertaking a very difficult issue, one in which they had to listen to passionate arguments, for and against, from a host of constituents, individuals, and organizations. Effective organizations such as the ADA cannot dodge difficult issues; it is what makes them effective. The AADSM likewise is an effective organization and had the courage to challenge the issue of additional education with the ADA.

Challenging the ADA, while important, is not enough. The AADSM has taken on the responsibility of providing the minimum required education for dentists desiring to enter the field of dental sleep medicine. In September 2018, the AADSM launched the AADSM Mastery Program. The program provides dentists with the minimum education required to safely and effectively treat patients with oral appliance therapy. Mastery consists of three levels of education. After completion of Mastery I, dentists earn the AADSM Qualified Dentist designation. The designation is awarded regardless of AADSM membership. I want to be clear the AADSM Mastery Program series is not a requirement, membership benefit, or a recruitment tool for members. It is about ensuring those dentists who desire to treat patients with OSA or snoring are properly trained so that their patients are safely and effectively treated. Dentists who choose and complete Mastery II and Mastery III are then eligible to sit for the American Board of Dental Sleep Medicine certification examination. Keep in mind this is a personal achievement, NOT a requirement to practice.

AADSM recognizes it cannot be the sole provider of the AADSM Mastery Program. As such, the AADSM has reached out to all US dental schools, inviting them to offer an accredited AADSM Mastery Program. Our sole purpose in doing this is to make necessary education available to dentists.

Many readers are no doubt asking why has the 
AADSM undertaken such an ambitious effort. The AADSM strongly believes a standardized education based on a core curriculum is necessary for all dentists treating patients with oral appliance therapy and is essential for the growth of the field. Further, it demonstrates to insurance companies, employers, referring sleep physicians and most importantly patients, that oral appliance therapy is a credible treatment and providers should be selected based on their qualifications.

Make no mistake, the role of dentists preventing medical diseases (e.g. providing immunizations) and treating illnesses such as OSA, snoring, type 2 diabetes, and hypertension is only in its infancy. The role of dentists will grow; it must and soon. The severe shortage of sleep physicians and primary care physicians is at a critical point, and it is only going to get worse. ${ }^{3}$ Nurse practitioners ${ }^{4}$ and physician assistants ${ }^{5}$ are facing similar shortages and are already stretched beyond their limits. The need for qualified trained medical providers must expand to include dentists. As we expand our role in health care, we have a responsibility to all affected by our expansion to demonstrate competency, and the only way to get there is through education. That is why we are calling on the ADA to amend its policy to include an educational requirement to treat patients in whom OSA and snoring have been diagnosed. In the meantime, we call on all dentists who are or plan to treat these patients with oral appliance therapy to attend the AADSM Mastery Program, whether it is offered by the AADSM or an accredited dental school.

Standardized education is only the first of many steps the AADSM will undertake over the next few years. If our vision to advance the field is to be realized, every dentist, regardless of where you practice, must not only think about the future, but start to prepare for it. Our professional lives are on the verge of change.

We can sit back and watch the future become the past, or we can take the reins and shape the future. I've decided where I will be; I hope you join me.

\section{CITATION}

Schwartz, D. The future belongs to those who shape it. J Dent Sleep Med. 2019;6(1)

\section{REFERENCES}

1. Schwartz DB. What does the ADA proposed policy on sleeprelated breathing disorders mean for dentists and patients? J Dent Sleep Med. 2017;04(02):27-28. doi:10.15331/jdsm.6520

2. American Dental Association. The Role of Dentistry in the Treatment of Sleep Related Breathing Disorders. https://www.ada.org/en/ /media/ADA/Member Center/FIles/TheRole-of-Dentistry-in-Sleep-Related-Breathing-Disorders. Published 2017. Accessed December 11, 2018.

3. Dall T, West T, Chakrabarti R, Reynolds R, Iacobucci W. 2018 Update: The complexities of physician supply and demand: projections from 2014 to 2025: Final Report. Assoc Am Med Coll. 2018;(March):1-51. doi:10.13140/RG.2.2.16013.95209

4. Sanborn BJ. Hospitals bracing for four-year nursing shortage by partnering with colleges | Healthcare Finance News. https://www.healthcarefinancenews.com/news/hospitals-bracingfour-year-nursing-shortage-partnering-colleges. March 12, 2018 Accessed December 11, 2018.

5. Projecting the Supply and Demand for Primary Care Practitioners Through 2020. Health Resources \& Services Administration. https://bhw.hrsa.gov/health-workforce-analysis/primary-care2020. November 2013. Accessed December 11, 2018.

\section{SUBMISSION AND CORRESPONDENCE INFORMATION}

\section{Submitted in final revised form September 27, 2018 Accepted for publication September 27, 2018}

Address correspondence to: David Schwartz, DDS, D.ABDSM, 9933 Lawler Ave, \#340A, Skokie, IL 60077, Email: drdavid@ drdavidschwartz.com

\section{DISCLOSURE STATEMENT}

Dr. Schwartz reports serving as part of an advisory group for Prosomnus and having a financial stake in Prosomnus. 